09

\title{
Critical dependence of the excitonic absorption in cuprous oxide on experimental parameters
}

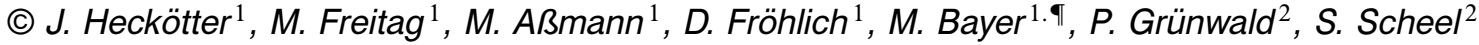 \\ ${ }^{1}$ Experimentelle Physik 2, Technische Universität Dortmund, \\ D-44221 Dortmund, Germany \\ ${ }^{2}$ Institut für Physik, Universität Rostock, Albert-Einstein-Strasse 23, \\ D-18059 Rostock, Germany \\ ๑ E-mail: manfred.bayer@tu-dortmund.de
}

We study the modification of the exciton absorption in cuprous oxide by the presence of free carriers excited through above band gap excitation. Without this pumping, the absorption spectrum below the band gap consists of the yellow exciton series with principal quantum numbers up to more than $n=20$, depending on the temperature, changing over to an about constant, only slowly varying absorption above the gap. Careful injection of free carriers, starting from densities well below $1 \mu \mathrm{m}^{-3}$, leads to a reduction of the band gap through correlation effects. The excitons in the Rydberg regime above $n=10$ remain unaffected until the band gap approaches them. Then they loose oscillator strength and ultimately vanish upon crossing with the band gap.

We gratefully acknowledge support by the Priority Programme 1929 „Giant Interactions in Rydberg Systems“, the TRR 160 „Coherent manipulation of interacting spin excitations in tailored semiconductors“", and the SFB 652/3 „Strong correlations in the radiation field“, all funded by the Deutsche Forschungsgemeinschaft. MB also acknowledges support by the RF Government Grant N 14.Z50.31.0021.

We would like to thank D. Semkat and H. Stolz for fruitful discussions.

DOI: $10.21883 /$ FTT.2018.08.46251.19Gr

* Полный текст статьи опубликован в журнале „Physics of the Solid State“ (Т. 60. Вып. 8). 